PROCEEDINGS OF THE

AMERICAN MATHEMATICAL SOCIETY

Volume 131, Number 6, Pages 1917-1923

S 0002-9939(02)06739-4

Article electronically published on September 20, 2002

\title{
APPROXIMATING SPECTRAL INVARIANTS OF HARPER OPERATORS ON GRAPHS II
}

\author{
VARGHESE MATHAI, THOMAS SCHICK, AND STUART YATES
}

(Communicated by Jozef Dodziuk)

\begin{abstract}
We study Harper operators and the closely related discrete magnetic Laplacians (DML) on a graph with a free action of a discrete group, as defined by Sunada. The spectral density function of the DML is defined using the von Neumann trace associated with the free action of a discrete group on a graph. The main result in this paper states that when the group is amenable, the spectral density function is equal to the integrated density of states of the DML that is defined using either Dirichlet or Neumann boundary conditions. This establishes the main conjecture in a paper by Mathai and Yates. The result is generalized to other self adjoint operators with finite propagation speed.
\end{abstract}

\section{INTRODUCTION}

In MY Mathai and Yates examined the spectral properties of the discrete magnetic Laplacian (DML) on a graph with a free action of a discrete group, as defined by Sunada [Sun]. One of the results proved in [MY] is that when the group is amenable and has a finite fundamental domain on the graph, the spectral density function of the DML - defined using the von Neumann trace associated with the discrete group action - can be approximated almost everywhere by the normalized spectral densities of a series of finite approximations of the DML, consisting of restrictions of the DML to a regular exhaustion of the graph. The latter approximation is known in the physics literature as the integrated density of states of the DML, so that the result can be rephrased as saying that the von Neumann spectral density function of the DML is equal to the integrated density of states of the DML almost everywhere. This partial result was known to the experts; cf. Bel, Sh].

It was shown in $[\mathrm{MY}]$ that in fact the approximation holds at every point whenever the DML is associated with a rational weight function, and it was conjectured there that the assumption on the weight function was irrelevant. Based on the idea of the second author, the conjecture is established here in complete generality, and at the same time a much more straightforward argument is presented for the corresponding result in [MY]. Related arguments can be found for example in [DLMSY],

Received by the editors January 12, 2002.

2000 Mathematics Subject Classification. Primary 58J50, 39A12.

Key words and phrases. Harper operator, discrete magnetic Laplacian, DML, approximation theorems, amenable groups, von Neumann algebras, graphs, integrated density of states.

The first and third authors acknowledge support from the Australian Research Council. 
Eck] and [El], and are known to other authors, in particular Wolfgang Lück and Holger Reich.

Hereafter, let $X$ be a combinatorial graph with finite fundamental domain $\mathcal{F}$ under the free action of an amenable discrete group $\Gamma$. Each edge $[e]$ of $X$ has associated with it two oriented edges $e$ and $\bar{e}$ with opposite orientation. While denoting the set of oriented edges by Edge $X$, it will be convenient to work with a subset $E^{+}$of these edges in which each combinatorial edge has exactly one oriented representative. Unless otherwise stated, functions over $X$ will refer to complexvalued functions over the vertices of $X$.

By Følner's characterization of amenability (see also [Ad]) there must exist a regular exhaustion of $\Gamma$ : a tower of finite subsets $\Lambda_{m} \subset \Lambda_{m+1}, \bigcup_{m} \Lambda_{m}=\Gamma$ satisfying

$$
\lim _{k \rightarrow \infty} \frac{\# \partial_{\delta} \Lambda_{k}}{\# \Lambda_{k}}=0 \quad \forall \delta>0
$$

where $\partial_{\delta} \Lambda_{k}$ denotes the $\delta$-neighborhood of the boundary of $\Lambda_{k}$ in $\Gamma$ in the word metric (with respect to some fixed generating set).

Let $\left\{X_{m}\right\}$ be a sequence of subgraphs of $X$, with $X_{m}$ being the largest subgraph of $X$ contained within the translates by $\Lambda_{m}$ of the fundamental domain. Then the $X_{m}$ form a regular exhaustion of the graph, satisfying

$$
\lim _{m \rightarrow \infty} \frac{\# \operatorname{Vert} \partial_{\delta} X_{m}}{\# \operatorname{Vert} X_{m}}=0 \quad \forall \delta>0
$$

where $\partial_{\delta} X_{m}$ refers to the subgraph which is the $\delta$-neighborhood of the boundary of $X_{m}$ in $X$ in the simplicial metric.

The discrete magnetic Laplacian $\Delta_{\sigma}$ is an example of an operator with bounded propagation speed, that is, there exists a constant $c$ such that for any function $f$ with support $Y \subset X$, the function $\Delta_{\sigma} f$ has support in the $c$-neighborhood of $Y$. The following theorem, proved in Section 3, provides an approximation for the spectral density function of such operators. This was posed (for the DML) as Conjecture 0.1 of $[\mathrm{MY}]$, where a proof was obtained for all points $\lambda$ only when the operator was the DML associated with a rational weight function $\sigma$.

Theorem 1.1 (Strong spectral approximation theorem). Let $X$ be a graph on which there is a free group action by an amenable group $\Gamma$, with finite fundamental domain. Let $\left\{X_{m}\right\}_{m=1}^{\infty}$ be the regular exhaustion of $X$ corresponding to a regular exhaustion $\Lambda_{m}$ of $\Gamma$. Let $A$ be a self-adjoint operator of bounded propagation speed acting on $l^{2}(X)$, which commutes with an adjoint-closed set of twisted translation operators and has spectral density function $F$. Construct finite approximations $A_{m}$ to $A$ by restricting $A$ to the space of functions supported on $X_{m}$, and denote by $F_{m}$ the normalized spectral density function of $A_{m}$,

$$
F_{m}(\lambda)=\frac{1}{\# \Lambda_{m}} \#\left\{\text { eigenvalues } \leq \lambda \text { of } A_{m} \text {, counting multiplicity }\right\} .
$$

Then

$$
\lim _{m \rightarrow \infty} F_{m}(\lambda)=F(\lambda), \quad \forall \lambda \in \mathbb{R} .
$$

\section{The Discrete MAGNETiC LAPLACiAN} AND OPERATORS OF BOUNDED PROPAGATION SPEED

The DML and the Harper operator are defined by a $U(1)$-valued weight function $\sigma$ on the edges of the graph $X$ which is weakly $\Gamma$-invariant. That is, for any edge 
$e, \sigma(\bar{e})=\overline{\sigma(e)}$, and for any $\gamma \in \Gamma$ there exists some $U(1)$-valued function $s_{\gamma}$ on the vertices of $X$ such that

$$
\sigma(\gamma e)=\sigma(e) s_{\gamma}(\mathfrak{t}(e)) \overline{s_{\gamma}(\mathfrak{o}(e))}
$$

where $\mathfrak{o}(e)$ and $\mathfrak{t}(e)$ are the origin and terminus of the edge $e$ respectively. The Harper operator $H_{\sigma}$ is then given by

$$
\left(H_{\sigma} f\right)(v):=\sum_{\substack{e \in E^{+} \\ \mathfrak{t}(e)=v}} \sigma(e) f(\mathfrak{o}(e))-\sum_{\substack{e \in E^{+} \\ \mathfrak{o}(e)=v}} \overline{\sigma(e)} f(\mathfrak{t}(e))
$$

and the DML $\Delta_{\sigma}$ is defined by

$$
\left(\Delta_{\sigma} f\right)(v):=\mathcal{O}(v) f(v)-\left(H_{\sigma} f\right)(v),
$$

where $\mathcal{O}(v)$ is the valence of the vertex $v$.

For some $s_{\gamma}$ satisfying the weak $\Gamma$-invariance property for $\sigma$ (2.1), one can define the magnetic translation operators $T_{\gamma}$,

$$
\left(T_{\gamma} f\right)(x)=s_{\gamma}\left(\gamma^{-1} x\right) f\left(\gamma^{-1} x\right)
$$

These form an adjoint-closed set of operators over the space $l^{2}(X)$ of $L^{2}$ functions on the vertices of $X$, and thus determine a von Neumann algebra $B\left(l^{2}(X)\right)^{T_{\Gamma}}$ consisting of all operators over $l^{2}(X)$ that commute with the $T_{\gamma}$. This von Neumann algebra has a finite trace $\operatorname{Tr}_{\Gamma}$, defined in (2.4). The DML is a self-adjoint operator in this von Neumann algebra, and so one can define its spectral density function $F$ by

$$
F(\lambda)=\operatorname{Tr}_{\Gamma} E(\lambda)
$$

where $E(\lambda)=\chi_{(-\infty, \lambda]}\left(\Delta_{\sigma}\right)$ is the spectral projection of the DML for the interval $(-\infty, \lambda]$.

More generally one can start with an adjoint-closed set of twisted translation operators $T_{\gamma}$ of the form (2.2), and then consider a self-adjoint bounded operator $A$ in the von Neumann algebra $B\left(l^{2}(X)\right)^{T_{\Gamma}}$ which — like the DML — has bounded propagation speed.

Definition 2.1. An operator $A$ has propagation speed bounded by $R$ if for any function $f \in l^{2}(X)$, the support of $A f$ is a subset of the $R$-neighborhood of the support of $f$ in the simplicial metric.

The operator $A$ is weakly $\Gamma$-equivariant if there exists a $\Gamma$-indexed set of functions $t_{\gamma}:$ Vert $X \rightarrow U(1)$ defining twisted translation operators $T_{\gamma}$ :

$$
\left(T_{\gamma} f\right)(x)=t_{\gamma}\left(\gamma^{-1} x\right) f\left(\gamma^{-1} x\right)
$$

such that $A$ commutes with $T_{\gamma}$ and $T_{\gamma}^{*}$ for all $\gamma \in \Gamma$. Then $A$ is an element of the associated von Neumann algebra $B\left(l^{2}(X)\right)^{T_{\Gamma}}$ with trace

$$
\operatorname{Tr}_{\Gamma}(A)=\sum_{v \in \mathcal{F}}\left\langle A \delta_{v}, \delta_{v}\right\rangle
$$

For each of the subgraphs $X_{m}$ one can define a finite operator $A_{m}$ by restricting $A$ to those functions with support on the vertices of $X_{m}$; explicitly, $A_{m}=P_{m} A i_{m}$ where $P_{m}$ is the orthogonal projection onto $l^{2}\left(X_{m}\right)$ and $i_{m}: l^{2}\left(X_{m}\right) \rightarrow l^{2}(X)$ is the canonical inclusion. We define the normalized spectral density functions of these restricted operators by

$$
F_{m}(\lambda)=\frac{1}{\# \Lambda_{m}} \#\left\{\text { eigenvalues } \leq \lambda \text { of } A_{m}, \text { counting multiplicity }\right\} .
$$


Part (i) of Theorem 2.6 of [MY] relates limits of these $F_{m}$ to the spectral density function $F$ of $A$ when $A$ is the discrete magnetic Laplacian. The argument deployed though extends trivially to bounded self-adjoint weakly $\Gamma$-equivariant operators of bounded propagation speed generally.

Theorem 2.2 (Weak spectral approximation theorem). Take $X$ to be a graph as above, and let $A$ be a bounded self-adjoint weakly $\Gamma$-equivariant operator over $l^{2}(X)$ of bounded propagation speed, with restrictions $A_{m}$ to the regular exhaustion $X_{m}$ of $X$. Define the following limits of the normalized spectral density functions,

$$
\begin{aligned}
\bar{F}(\lambda) & =\limsup _{m \rightarrow \infty} F_{m}(\lambda), \\
\underline{F}(\lambda) & =\liminf _{m \rightarrow \infty} F_{m}(\lambda), \\
\bar{F}^{+}(\lambda) & =\lim _{\delta \rightarrow+0} \bar{F}(\lambda+\delta), \\
\underline{F}^{+}(\lambda) & =\lim _{\delta \rightarrow+0} \underline{F}(\lambda+\delta) .
\end{aligned}
$$

Then

$$
F(\lambda)=\bar{F}^{+}(\lambda)=\underline{F}^{+}(\lambda), \quad \forall \lambda \in \mathbb{R} .
$$

Proof. Refer to the proof of Theorem 2.6 of [MY].

As a direct consequence, one has that at every point $\lambda$ at which $F$ is continuous, that is, for every $\lambda \notin \operatorname{spec}_{\text {point }} A$,

$$
\lim _{m \rightarrow \infty} F_{m}(\lambda)=F(\lambda) .
$$

In particular, (2.8) holds for all but at most a countable set of points. In the following section a new argument is presented that extends this result to all $\lambda$.

\section{The Strong SPECTRAL APproximation RESUlt}

A consequence of Theorem 2.2 is that if the jump of $F$ at a discontinuity $\lambda$ can be approximated by the jumps at $\lambda$ of the piece-wise constant normalized spectral density functions $F_{m}$, then the limit of $F_{m}(\lambda)$ exists and equals $F(\lambda)$.

We first need an elementary lemma, whose proof is omitted.

Lemma 3.1. Given two sequences $\left\{a_{i}\right\}$ and $\left\{b_{i}\right\}$ with $a_{i} \leq b_{i}$ for all $i$, it follows that $\sup _{i} a_{i} \leq \sup _{i} b_{i}$ and $\inf _{i} a_{i} \leq \inf _{i} b_{i}$.

In particular, if $\left\{f_{i}\right\}$ is a sequence of monotonically increasing functions, then $\liminf _{i \rightarrow \infty} f_{i}$ and $\limsup _{i \rightarrow \infty} f_{i}$ are also monotonically increasing.

The corollary then follows from Theorem 2.2

Corollary 3.2. Denote by $D(\lambda)$ the jump in $F$ at $\lambda$,

$$
D(\lambda)=\lim _{\delta \rightarrow+0} F(\lambda)-F(\lambda-\delta),
$$

and similarly let $D_{m}(\lambda)$ be the jump in the normalized spectral density functions $F_{m}$

$$
D_{m}(\lambda)=\lim _{\delta \rightarrow+0} F_{m}(\lambda)-F_{m}(\lambda-\delta)
$$

Then for any $\lambda \in \mathbb{R}$,

$$
\lim _{m \rightarrow \infty} D_{m}(\lambda)=D(\lambda) \Longrightarrow \lim _{m \rightarrow \infty} F_{m}(\lambda)=F(\lambda) .
$$


Proof. Fix some $\epsilon>0$ and $\lambda \in \mathbb{R}$, and suppose that $\lim _{m \rightarrow \infty} D_{m}(\lambda)=D(\lambda)$. Then we will show that $F(\lambda)-3 \epsilon<F_{m}(\lambda)<F(\lambda)+\epsilon$ for all sufficiently large $m$.

By the right continuity and monotonicity of $F$, there exists a $\delta>0$ such that $F(x)<F(\lambda)+\frac{\epsilon}{2}$ for all $\lambda \leq x<\lambda+\delta$. The $F_{m}$ approach $F$ at all points of continuity of $F$; as $F$ has at most a countable number of discontinuities there exists some $x_{0} \in[\lambda, \lambda+\delta)$ where $\lim _{m \rightarrow \infty} F_{m}\left(x_{0}\right)=F\left(x_{0}\right)<F(\lambda)+\frac{\epsilon}{2}$. So for $m$ greater than some $M_{1}$, it follows that $F_{m}\left(x_{0}\right)<F(\lambda)+\epsilon$. The $F_{m}$ are monotonically increasing, so

$$
\exists M_{1} \text { such that } F_{m}(\lambda)<F(\lambda)+\epsilon \quad \forall m>M_{1} .
$$

Now $F(\lambda)=D(\lambda)+\lim _{\delta \rightarrow+0} F(\lambda-\delta)$, so we can choose $d$ such that $F(\lambda-d)+$ $D(\lambda)>F(\lambda)-\epsilon$. As the $F_{m}$ are monotonically increasing functions, $\underline{F}$ is also by Lemma 3.1. By Theorem 2.2, $\underline{F}^{+}(\mu)=F(\mu)$ for all $\mu$. Therefore $\underline{F}(\mu+\delta)>F(\mu)$ for all $\delta>0$. Letting $\mu=\lambda-d$ and $\delta=\frac{d}{2}$ gives $\underline{F}\left(\lambda-\frac{d}{2}\right)>F(\lambda-d)$ and so for sufficiently large $m, F_{m}\left(\lambda-\frac{d}{2}\right)>F(\lambda-d)-\epsilon$.

From $F_{m}(\lambda) \geq F_{m}\left(\lambda-\frac{d}{2}\right)+D_{m}(\lambda)$ and $F(\lambda-d)>F(\lambda)-D(\lambda)-\epsilon$,

$$
\exists M_{2} \text { such that } \quad \begin{aligned}
F_{m}(\lambda) & >F(\lambda-d)-\epsilon+D_{m}(\lambda) \\
& >F(\lambda)+D_{m}(\lambda)-D(\lambda)-2 \epsilon \\
& \geq F(\lambda)-\left|D_{m}(\lambda)-D(\lambda)\right|-2 \epsilon \quad \forall m>M_{2} .
\end{aligned}
$$

By supposition, $\lim _{m \rightarrow \infty} D_{m}(\lambda)=D(\lambda)$. In particular we can pick an $M$ greater than $M_{1}$ and $M_{2}$ such that $\left|D_{m}(\lambda)-D(\lambda)\right|<\epsilon$ for all $m>M$. Therefore

$$
\exists M \text { such that } m>M \Longrightarrow F(\lambda)+\epsilon>F_{m}(\lambda)>F(\lambda)-3 \epsilon,
$$

and thus

$$
\lim _{m \rightarrow \infty} D_{m}(\lambda)=D(\lambda) \Longrightarrow \lim _{m \rightarrow \infty} F_{m}(\lambda)=F(\lambda)
$$

The following theorem establishes this approximation of the jumps. Its proof is modelled on the argument in [El].

Theorem 3.3. The jump $D(\lambda)$ of $F$ at $\lambda$ is the limit of the jumps of the normalized spectral density functions:

$$
\lim _{m \rightarrow \infty} D_{m}(\lambda)=D(\lambda) \quad \forall \lambda \in \mathbb{R} .
$$

Proof. Note that the jumps can be expressed in terms of the dimensions of kernels,

$$
\begin{aligned}
D(\lambda) & =\operatorname{dim}_{\Gamma} \operatorname{ker}(A-\lambda), \\
D_{m}(\lambda) & =\frac{1}{\# \Lambda_{m}} \operatorname{dim} \operatorname{ker}\left(A_{m}-\lambda\right) .
\end{aligned}
$$

Consider subsets $Y_{m}$ of $X_{m}$ consisting of the $r$-interior of $X_{m}$, where $A$ has propagation speed bounded by $r$.

For each $X_{m}$ define $\operatorname{dim}_{X_{k}}$ for a subspace $W$ of $l^{2}(X)$ by

$$
\operatorname{dim}_{X_{k}} W=\frac{1}{\# \Lambda_{k}} \sum_{x \in X_{k}}\left\langle P_{W} \delta_{x}, \delta_{x}\right\rangle
$$


where $P_{W}$ is the orthogonal projection onto $W$. This has the following properties for subspaces $W, V$ of $l^{2}(X)$ :

(1) $W \perp V \Longrightarrow \operatorname{dim}_{X_{k}}(W \oplus V)=\operatorname{dim}_{X_{k}} W+\operatorname{dim}_{X_{k}} V$,

(2) $W \subset V \Longrightarrow \operatorname{dim}_{X_{k}} W \leq \operatorname{dim}_{X_{k}} V$,

(3) $P_{W} \in B\left(l^{2}(X)\right)^{T_{\Gamma}} \Longrightarrow \operatorname{dim}_{X_{k}} W=\operatorname{dim}_{\Gamma} W$,

(4) $W \subset l^{2}\left(X_{k}\right) \Longrightarrow \operatorname{dim}_{X_{k}} W=\frac{1}{\# \Lambda_{k}} \operatorname{dim} W$, regarding $l^{2}\left(X_{k}\right)$ as a subspace of $l^{2}(X)$.

In particular, $\operatorname{dim}_{X_{k}} l^{2}\left(X_{k}\right)=\# \mathcal{F}$ and $\operatorname{dim}_{X_{k}} l^{2}\left(Y_{k}\right)$ converges to $\# \mathcal{F}$ as $k$ approaches infinity.

Let $i_{m}^{\prime}: l^{2}\left(Y_{m}\right) \rightarrow l^{2}\left(X_{m}\right)$ be the inclusion with $\left(i_{m}^{\prime} f\right)(x)=0$ for all $x$ in $X_{m} \backslash Y_{m}$. Let $A_{m}^{\prime}: l^{2}\left(Y_{m}\right) \rightarrow l^{2}\left(X_{m}\right)$ be the operator $A_{m} i_{m}^{\prime}$. For any function $f$ in $l^{2}\left(Y_{m}\right)$, $\left(A_{m}^{\prime}-\lambda i_{m}^{\prime}\right) f=\left(A_{m}-\lambda\right)\left(i_{m}^{\prime} f\right)$. Therefore as subspaces of $l^{2}(X)$,

$$
\begin{gathered}
\operatorname{ker}\left(A_{k}^{\prime}-\lambda i_{k}^{\prime}\right) \subset \operatorname{ker}\left(A_{k}-\lambda\right), \\
\operatorname{im}\left(A_{k}^{\prime}-\lambda i_{k}^{\prime}\right) \subset \operatorname{im}\left(A_{k}-\lambda\right) .
\end{gathered}
$$

Let $D_{k}^{\prime}(\lambda)=\frac{1}{\# \Lambda_{m}} \operatorname{dim} \operatorname{ker}\left(A_{k}^{\prime}-\lambda i_{k}^{\prime}\right)$. Then taking $\operatorname{dim}_{X_{k}}$,

$$
\begin{aligned}
D_{k}^{\prime}(\lambda)= & \operatorname{dim}_{X_{k}} \operatorname{ker}\left(A_{k}^{\prime}-\lambda i_{k}^{\prime}\right) \leq \operatorname{dim}_{X_{k}} \operatorname{ker}\left(A_{k}-\lambda\right)=D_{k}(\lambda), \\
& \operatorname{dim}_{X_{k}} \operatorname{im}\left(A_{k}^{\prime}-\lambda i_{k}^{\prime}\right) \leq \operatorname{dim}_{X_{k}} \operatorname{im}\left(A_{k}-\lambda\right) .
\end{aligned}
$$

One also has for any $m$,

$$
\begin{aligned}
\lim _{k \rightarrow \infty} \operatorname{dim}_{X_{k}} \operatorname{ker}\left(A_{k}^{\prime}-\lambda i_{k}^{\prime}\right) & +\operatorname{dim}_{X_{k}} \operatorname{im}\left(A_{k}^{\prime}-\lambda i_{k}^{\prime}\right)=\lim _{k \rightarrow \infty} \operatorname{dim}_{X_{k}} l^{2}\left(Y_{k}\right) \\
& =\# \mathcal{F}=\operatorname{dim}_{X_{m}} \operatorname{ker}\left(A_{m}-\lambda\right)+\operatorname{dim}_{X_{m}} \operatorname{im}\left(A_{m}-\lambda\right) .
\end{aligned}
$$

Consequently,

$$
\lim _{k \rightarrow \infty} D_{k}(\lambda)-D_{k}^{\prime}(\lambda)=0
$$

Recall that $A_{k}=P_{k} A i_{k}$, where $P_{k}$ is the projection onto $l^{2}\left(X_{k}\right)$ and $i_{k}$ is the canonical inclusion of $l^{2}\left(X_{k}\right)$ into $l^{2}(X)$. In terms of $A$ then, $A_{k}^{\prime}=P_{k} A i_{k} i_{k}^{\prime}$. By the bounded propagation speed of $A$, the support of $A i_{k} i_{k}^{\prime} f$ is contained within $X_{k}$ for any $f$ in $l^{2}\left(Y_{k}\right)$ and hence as in (3.5),

$$
\begin{aligned}
& \operatorname{ker}\left(A_{k}^{\prime}-\lambda i_{k}^{\prime}\right) \subset \operatorname{ker}(A-\lambda), \\
& \operatorname{im}\left(A_{k}^{\prime}-\lambda i_{k}^{\prime}\right) \subset \operatorname{im}(A-\lambda) .
\end{aligned}
$$

The kernel and image of $A-\lambda$ are invariant under the twisted translations $T_{\gamma}$, and so for these subspaces, $\operatorname{dim}_{X_{k}}$ equals $\operatorname{dim}_{\Gamma}$. It follows that

$$
\begin{aligned}
D_{k}^{\prime}(\lambda)= & \operatorname{dim}_{X_{k}} \operatorname{ker}\left(A_{k}^{\prime}-\lambda i_{k}^{\prime}\right) \leq \operatorname{dim}_{\Gamma} \operatorname{ker}\left(A_{k}-\lambda\right)=D(\lambda), \\
& \operatorname{dim}_{X_{k}} \operatorname{im}\left(A_{k}^{\prime}-\lambda i_{k}^{\prime}\right) \leq \operatorname{dim}_{\Gamma} \operatorname{im}\left(A_{k}-\lambda\right),
\end{aligned}
$$

and

$$
\begin{aligned}
\lim _{k \rightarrow \infty} \operatorname{dim}_{X_{k}} \operatorname{ker}\left(A_{k}^{\prime}-\lambda i_{k}^{\prime}\right)+\operatorname{dim}_{X_{k}} \operatorname{im}\left(A_{k}^{\prime}-\lambda i_{k}^{\prime}\right) \\
=\# \mathcal{F}=\operatorname{dim}_{\Gamma} \operatorname{ker}(A-\lambda)+\operatorname{dim}_{\Gamma} \operatorname{im}(A-\lambda) .
\end{aligned}
$$


Therefore

$$
\lim _{k \rightarrow \infty} D_{k}^{\prime}(\lambda)=D(\lambda)
$$

which together with (3.6) gives the required limit

$$
\lim _{k \rightarrow \infty} D_{k}(\lambda)=D(\lambda) .
$$

Theorem 1.1 follows from Theorem 3.3 and Corollary 3.2 .

\section{REFERENCES}

[Ad] T. Adachi, A note on the Følner condition of amenability, Nagoya Math. J. 131 (1993) 67-74. MR 94h:43001

[Bel] J. Bellissard, Gap Labelling Theorems for Schrödinger's Operators, From number theory to physics (Les Houches, 1989), 538-630, Springer, Berlin, 1992. MR 94e:46120

[DLMSY] J. Dodziuk, P. Linnell, V. Mathai, T. Schick and S. Yates, Approximating $L^{2}$-invariants, and the Atiyah conjecture, Commun. in Pure and Applied Math. (to appear).

[Eck] B. Eckmann, Approximating $\ell_{2}$-Betti numbers of an amenable covering by ordinary Betti numbers, Comment. Math. Helv. 74 (1999) 150-155. MR 2000c:57050

[El] G. Elek, On the analytic zero divisor conjecture of Linnell, math.GR/0111180.

[MY] V. Mathai and S. Yates, Approximating spectral invariants of Harper operators on graphs, J. Functional Analysis 188 (2002), no. 1, 111-136.

[Sh] M. Shubin, Discrete Magnetic Laplacian, Commun. Math. Phys. 164 (1994), no. 2, 259-275. MR 97b:82010

[Sun] T. Sunada, A discrete analogue of periodic magnetic Schrödinger operators, Contemp. Math. 173 (1994), 283-299. MR 95i:58185

Department of Mathematics, University of Adelaide, Adelaide 5005, Australia

E-mail address: vmathai@maths.adelaide.edu.au

FB Mathematik, Universität Göttingen, Bunsenstrasse 3, 37073 Göttingen, Germany

E-mail address: schick@uni-math.gwdg.de

Department of Mathematics, University of Adelaide, Adelaide 5005, Australia

E-mail address: syates@maths.adelaide.edu.au 